\title{
Formulation and in vitro Evaluation of Betahistine Dihydrochloride Twice Daily Controlled Release Matrix Tablet
}

\author{
Asraful Islam, Shimul Halder and Sitesh Chandra Bachar
}

Department of Pharmaceutical Technology,University of Dhaka,Dhaka-1000, Bangladesh

\begin{abstract}
In order to reduce dosing frequency, a sustained release dosage form of betahistine dihydrochloride was developed as a twice daily controlled release tablet formulation that could be used to decrease vertigo resulted in Meniere's disease for a prolonged time. Six formulations were developed by using Methocel K4M CR, Methocel K15M CR and Methocel K 100 LVCR as single and combinations in different percentage. The tablets were prepared by wet granulation method. Physical properties of betahistine dihydrochloride granules and betahistine dihydrochloride matrix tablets were evaluated. The tablets were characterized for Betahistine Dihydrochloride release in both gastric simulated fluid $(0.1 \mathrm{~N} \mathrm{HCl}, \mathrm{pH} 1.3)$ and simulated intestinal fluid (buffer solution $\mathrm{pH}$ 6.8). The data were subjected to different models in order to evaluate their release kinetics and mechanisms. The results of the in vitro dissolution study indicate that most of the formulations showed above $80 \%$ of drug release in 12 hours but formulations (F-2 and F-4) met the official specification of release profile. Dissolution data were fitted to zero order equation, Higuchi square root law, Korsmeyer-Peppas model and Hixson-Crowell cube root law as these plots showed the highest value of linearity to evaluate kinetic data. The release of betahistine dihydrochloride was prolonged for 12 hours indicating the usefulness of the formulations for twice daily dosage forms, leading to improve efficacy, controlling the release and better patient compliance.
\end{abstract}

Key words: Betahistine Dihydrochloride, Methocel K4M CR, Methocel K15M CR, Methocel K 100 LVCR, Controlled release, Meniere's disease

\section{INTRODUCTION}

During the past 30 years, as expenses and complications involved in marketing new drug molecules have increased with concomitant recognition of therapeutic advantages of controlled drug delivery, greater attention has been focused on the development of controlled release drug delivery systems (CRDDS). The goal in designing CRDDS is to reduce the frequency of dosing or to increase the effectiveness of the drug by localization at the site of action reducing the dose required or providing uniform drug delivery. ${ }^{1}$ The use of controlled release (CR) formulations offer many potential advantages, such as sustained blood levels, attenuation of adverse effects and improved patient compliance.

Correspondence to: Shimul Halder Cell: 01716599577

E-mail: sk_halder_85@yahoo.com

Dhaka Univ. J. Pharm. Sci. 10(2): 93-100, 2011 (December)
Betahistine is a histamine analog that was developed following successful parenteral use of histamine in patients with Meniere's disease. Betahistine relieves vertigo symptoms by improving circulation in the microvasculature of the inner ear which leads to a pressure reduction on the membranous labyrinth and relieves the symptoms of Meniere's disease. ${ }^{2}$ Betahistine Dihydrochloride is Nmethyl-2-(pyridin-2-yl) ethanamine, with chemical formula $\mathrm{C}_{8} \mathrm{H}_{12} \mathrm{~N}_{2}$, and has a molecular weight of 136.194. The successful treatment of vertigo depends on the maintenance of effective drug concentration level in the body for which a constant and uniform supply of drug is desired. The shorter biological half life (3-4 hours), rapidly and completely absorption nature and the dosage frequency three times a day make Betahistine Dihydrochloride an ideal candidate for sustained release formulations, which reduce the frequency of dose in order to improve patient 
compliance. The most common method of modulating drug release is to include it in a matrix system. Because of their flexibility, hydrophilic polymer matrix systems are widely used in oral controlled drug delivery to obtain a desirable drug release profile 3 , cost effectiveness, and broad regulatory acceptance. Among the hydrophilic polymers, hydroxypropyl methyl cellulose derivatives are frequently used because of its nontoxic nature, easy compression, swelling properties and accommodation to high levels of drug loading. ${ }^{4}$ Additionally, HPMC is a $\mathrm{pH}$ independent material and hence drug release from hydroxypropyl methyl cellulose matrix formulations is generally independent of processing variables. ${ }^{3,5}$ Wet granulation technique was effective mode of preparation as the dose is quite low $(12 \mathrm{mg})$ to ensure dose uniformity.

They are suitable for preparing formulations with soluble or insoluble drugs and at high or low dosage levels. Hydration of polymers results in the formation of a gel layer that controls the release rate of drug from the core of matrix tablets. The permeability of drug through Methocel K4M CR and/or K15M CR is independent of the $\mathrm{pH}$ of the digestive tract. Soluble drugs are released by the combination of diffusion and erosion mechanisms whereas erosion is the predominant mechanism for insoluble drugs. ${ }^{6}$ As Methocel derivatives are highly hydrophilic in nature, the involvement of water or moist granulation can make the process highly problematic, therefore, a dry process that produces acceptable powder characteristics and does not intervene with drug release characteristics would be desirable.

The aim of this work was to prepare uncoated hydrophilic matrix tablets containing Betahistine Dihydrochloride as a model drug, Methocel K4M CR, Methocel K15M CR and Methocel K 100LVCR in different ratios as hydrophilic matrix to retard drug release. Moreover, it was desirable to develop a formulation that will improve the bioavailability as well as control the release of Betahistine dihydrochloride ${ }^{7}$. Another objective of this work was to evaluate drug release data using various kinetic models in order to determine the mechanism of drug release.

\section{MATERIALS AND METHODS}

Materials. The following materials were used Betahistine Dihydrochloride (Cipla, India, potency: 99.01\%) Methocel K4M CR, Methocel K15M CR, Methocel K100 LVCR (Colorcon), Microcystalline Cellulose (Avicel-101) (Zhejiang Tianxin Pharmaceuticals Ltd.) Colloidal Anhydrous Silica (Aerosil 200) (Hanau Chemicals Ltd.), Talc (Sri Krishna Pharmaceuticals Ltd.)

Solvents and reagents. Citric Acid, Distilled water, Karl Fischer Reagent.

Equipments. Rapid Mix Granulator, \# 30 Mesh Screen, Rotary Tablet Punch (8 station), Tablet Hardness Tester (YD-1 hardness tester), Friability Tester (Electrolab EF-1), Electrolab Tablet Dissolution Test Machine (08L), Electronic Balance, Double Beam UV Spectrophotometer, Karl Fischer Instrument, Tap Density Tester.

Preparation of matrix tablet. Drug, polymers and other excipients were weighed separately for 160 tablets for each formulation. The proposed formulations (as mentioned in Table 1) were coded as F-1, F-2, F-3, F-4, F-5 \& F-6. The tablets were prepared by wet granulation technology. Active ingredient (Betahistine Dihydrochloride), Methocel polymers (Methocel K15M CR/ Methocel K 4M CR and Methocel K100LV CR), Avicel and Citric Acid was blended in a polybag for 10 minutes. Then few amount of distilled water was added to it and the mix was subjected to Rapid Mix Granulator for preparation of granules.Then the wet granules were subjected to dry through an oven at $60^{\circ} \mathrm{C}$ temperature at 1 hour. The granules were passed through \# 30 sieves. After that Colloidal Silicon Dioxide (Aerosil 200) and talc was added to mix with it in a polybag for five minutes. The final granule mix was then compressed in an eight (8) station Rotary tablet Punch Machine equipped with $6.5 \mathrm{~mm}$ round punch and die set. After compression, all the preparations were stored in double polythene bags at room temperature for further study. 
Evaluation of physical properties of formulation granules. Bulk density: Both LBD (Loose Bulk Density) and TBD (Tapped Bulk Density) were determined by taking 2 gm of granules from each proposed formulations, previously lightly shaken to break any agglomerates formed, was introduced into a $10 \mathrm{ml}$ measuring cylinder. After the initial volume was observed, the cylinder was placed into the tap density tester and the machine was set to a fixed rpm. The reading of tapping was continued until no further change in volume was noted. Using the following equations LBD and TBD were calculated

Table 1. Formulation of betahistine dihydrochloride tablets (F-1 to F-6)

\begin{tabular}{|c|c|c|c|c|c|c|}
\hline \multirow{2}{*}{ Ingredients } & \multicolumn{6}{|c|}{ Formulations } \\
\hline & $\mathrm{F}-1$ & $\mathrm{~F}-2$ & $\mathrm{~F}-3$ & $\mathrm{~F}-4$ & $\mathrm{~F}-5$ & F-6 \\
\hline Betahistine Dihydrochloride & 12 & 12 & 12 & 12 & 12 & 12 \\
\hline Methocel K4M CR & 0 & 0 & 0 & 11.625 & 23.25 & 34.875 \\
\hline Methocel K15M CR & 23.25 & 11.625 & 34.875 & 0 & 0 & 0 \\
\hline Methocel K100 LVCR & 23.25 & 34.875 & 11.625 & 34.875 & 23.25 & 11.625 \\
\hline Avicel PH 101 & 33 & 33 & 33 & 33 & 33 & 33 \\
\hline Aerosil 200 & 0.5 & 0.5 & 0.5 & 0.5 & 0.5 & 0.5 \\
\hline Talc & 5.75 & 5.75 & 5.75 & 5.75 & 5.75 & 5.75 \\
\hline Citric Acid & 2.25 & 2.25 & 2.25 & 2.25 & 2.25 & 2.25 \\
\hline Final Weight (mg) & 100 & 100 & 100 & 100 & 100 & 100 \\
\hline
\end{tabular}

LBD $=$ Weight of the powder/Volume of the packing.

TBD $=$ Weight of the powder/Tapped volume of the packing.

Compressibility index. The compressibility index of the granules was determined by Carr's compressibility index:

Carr's index $(\%)=[(\mathrm{TBD}-\mathrm{LBD}) \mathrm{X} 100] / \mathrm{TBD}$

Total porosity. Total porosity was determined by measuring the volume occupied by a selected weight of powder (Vbulk) and the true volume of granules (the space occupied by the powder exclusive of spaces greater than the intermolecular space (V).

Porosity $(\%)=($ Vbulk - V) $/$ V bulk $\times 100$

Angle of repose. The angle of repose of granules was determined by the funnel method. Accurately weighed granules were taken in a funnel. The height of the funnel was adjusted in such a way that the tip of the funnel just touched the apex of the heap of the granules. The granules were allowed to flow through the funnel freely onto the surface. The diameter of the powder cone was measured and angle of repose was calculated using the following equation:

Angle of repose, $\theta=\tan -1(\mathrm{~h} / \mathrm{r})$

Where, $\mathrm{h}=$ Height in $\mathrm{cm}$ of the powder cone, $\mathrm{r}=$ Radius in cm of the powder cone.

Moisture content. Moisture content of granules was determined using Mettler Karl Fischer Titrator. About $120 \mathrm{mg}$ granules was weighed and added into the reagent solutions of the instrument, which was stirred and the tare weight was fed into the instrument. Then after certain duration of time the moisture content as $\% \mathrm{w} / \mathrm{w}$ was read on the monitor.

Flow properties. It is very important parameter to be measured since it affects the mass of uniformity of the dose. It is usually predicted from Hausner ratio and angle of repose measurement.

Hausner Ratio $=$ Tapped Density/Bulk Density

Evaluation of physical properties of Betahistine Dihydrochloride matrix tablets

Weight variation test. To study weight variation, 20 tablets from each formulation were weighed using an electronic balance and the test was performed according to the British Pharmacopeia. 
Hardness. For each formulation, the hardness of 5 tablets was determined using tablet hardness tester.

Friability. Friability of 5 tablets of each proposed formulations were determined using the Friability tester.

Surface area. As the shape of the tablet was round flat, so it can be compared with a cylinder.

Surface area of a cylinder is calculated by using the following equation:

Cylinder surface area $=\pi \mathrm{r} 2 \mathrm{~h}$

Where, $\mathrm{r}=$ Radius in $\mathrm{mm}$ of tablet and $\mathrm{h}=$ thickness in $\mathrm{mm}$ of the tablet.

In vitro release studies of Betahistine Dihydrochloride matrix tablets. In vitro drug release studies of the matrix tablets were carried out using a six-station USP XXII type II dissolution test apparatus (Eurolab, Germany) at $37^{\circ} \mathrm{C}\left( \pm 0.5^{\circ} \mathrm{C}\right)$ and $50 \mathrm{rpm}$ speed in $900 \mathrm{ml}$ of $0.1 \mathrm{~N}$ hydrochloric acid (gastric simulated fluid, $\mathrm{pH}$ 1.3) as a dissolution medium for first 2 hours and next 10 hours in intestinal simulated fluid (buffer solution, $\mathrm{pH}$ 6.8).

Acid stage. The medium simulating gastric fluid was prepared by diluting $11.4 \mathrm{ml}$ of Hydrochloric acid $(32 \%, w / v)$ with sufficient distilled water to make the volume $1000 \mathrm{ml} .900 \mathrm{ml}$ of $0.1 \mathrm{~N} \mathrm{HCl}$ was placed in each vessel and the apparatus was assembled. Six tablets from one formulation were weighed and placed in the baskets. $10 \mathrm{ml}$ samples were taken by filtration at regular time intervals (after each 1 hour) over a period of 2 hours. After each sampling, the volume of the dissolution medium was replaced with $10 \mathrm{ml}$ of dissolution medium. The absorbance of the samples, after filtration, was measured with a single-beam UV-spectrophotometer (Shimadzu UV-1601, Japan) at $261 \mathrm{~nm}$ and the amount of drug calculated. The release studies were conducted in triplicate, and the mean values of the percentage of drug released were plotted versus time.

Buffer stage. After 2 hours operation in the acid stage, $20 \mathrm{ml} \mathrm{NaOH}(25 \%)$ was added to the previous fluid. The $\mathrm{pH}(6.8 \pm 0.05)$ was adjusted with the addition of $1.2 \mathrm{ml}$ ortho-phosphoric acid. The operation was continued for 10 hours. After each one hour interval $10 \mathrm{ml}$ of dissolution solution was sampled and the released drug assayed by using UV spectrophotometer (Shimadzu UV-1601, Japan) at $261 \mathrm{~nm}$. At each withdrawal $10 \mathrm{ml}$ of fresh dissolution medium was added. The dissolution study was continued for 10 hours to get a simulated picture of the drug release and the percentage of drug release was plotted against time.

Kinetic analysis of release data and mechanism of drug release. In order to evaluate the kinetics and the mechanism of drug release from the formulations, the data obtained from the in vitro drug release studies were analyzed by zero order, Higuchi and the Korsmeyer-Peppas models. To determine the mechanism of drug release from the formulations the data were treated according to zero-order (cumulative percent of drug released vs time, (Eq 1) the Higuchi cumulative percent of drug released vs square root of time, (SQRT), (Eq 2) models.

$$
\begin{aligned}
& M_{t}=M_{0}+k_{0} t-1-12 \\
& M_{t}=M_{0}+k_{H} t^{1 / 2}
\end{aligned}
$$

Where $\mathrm{M}_{\mathrm{t}}$ is the cumulative amount of drug released at any time, $t$ and $M_{0}$ are the dose of the drug incorporated in the delivery system and $k_{0}$ and $k_{H}$ are the rate constants for zero-order and Higuchi models, respectively.

The dissolution data were also fitted according to the well-known exponential equation of Peppas et al (Eq 3) which is often used to describe drug release behavior from polymeric systems.

$$
M_{t} / M_{\infty}=K t^{n}
$$

Where, $M_{t} / M_{\infty}$ is the fraction of drug released at time, $t, k$ is the kinetic constant, and $n$ is the diffusional exponent for drug release. The diffusional exponent, $n$, is dependent on the geometry of the device as well as the physical mechanism for release. A value of $\mathrm{n}=0.45$ indicates Fickian (case I) release; $>0.45$ but $<0.89$ for non-Fickian (anomalous) release; and $>0.89$ indicates super case II type of release. Case II generally refers to the erosion of the polymeric chain and anomalous transport (NonFickian) refers to a combination of both diffusion and erosion controlled drug release.

Statistical analysis. Data from the experiments were analyzed using the Statistical Package for Social 
Science (SPSS) software for windows version 17 (SPSS Inc., Chicago, Illinois, USA). Statistical analysis of the results was performed by using oneway analysis of variance (ANOVA) followed by Dennett's t-test for comparisons. The limit of significance was set at $\mathrm{p}<0.05$.

\section{RESULTS AND DISCUSSION}

The tablets of different formulations were subjected to various evaluation tests, such as weight variation, friability, hardness, thickness and content uniformity according to procedure specified in British Pharmacopoeia. the weight variation of the tablets from all batches varied between $0.5-2.1 \%$ $(1.17+0.56 \%)$,thickness between 3.86-3.97 mm $(3.91+0.05 \mathrm{~mm})$ and hardness $36.7-39.5 \mathrm{~N}(38.1+$ $0.7 \mathrm{~N}$ ) as shown in table 2. Friability of tablets fro different batches showed below $1 \%$. Good uniformity in drug content was found among different batches of the tablets and drug content was more than $95 \%$. The particle size of the blend was on an average of 420 Um and flow property was determined by Hausner ratio (1.18-1.22) and Carr's index (15.48-17.72) .hardness and disintegration time of all tablets from different batches shown to be satisfactory. All tablets found satisfactory release profile (Table 4).

Effect of polymers on drug release. The cumulative percent of Betahistine Dihydrochloride release from the matrix tablets of different batches were calculated by Zero order, First order and Higuchi Plot (Fig). The data showed that the release of drug from the prepared matrix tablets was depended on the type and amount of polymers. The best results were obtained in the case of formulas (F2, F-4 and F-6). Where different types of HPMCs (Both high viscosity grade and low viscosity grade) were used. Incase of increased amount of high viscosity grade HPMC leads to a delay in the release rate of active substances (F6) the release rates attained a value of more than $50 \%$ in $8^{\text {th }}$ when low viscosity grade HPMC constituted at least $34.5 \%$ of the matrix tablet .

But increase of low viscosity grades HPMC quantity by $50 \%$ didn't Trans late into a significant delay in the release of the active substance. The tablets of formulation F-1 and F-6 showed prolonged dissolution rate containing increased amount of HPMC with higher viscosity grades. Therefore the ratio of the polymers were seen to have a significant impact on the rate of drug release.

Release kinetics. Based on pharmacokinetics the release of profiles of the formulated tablets were compared with the theoretical sustained release heeded to select the optimized formulation. To know the mechanism of drug release from these formulations, the data were treated according to zero order (cumulative amount of drag released versus time) equations along with korsmeyer's (log cumulative parentages of in released versus log time) equations. The release rate kinetics. The Betahistine Dihydrochloride phosphate release rate kinetics was determined by multiple coefficients $\left(R^{2}\right)$ for individual formulation (Table 5). From the correlation coefficient values, it appears that Higuchi model is the best-fitting model for Betahistine Dihydrochloride phosphate release since higher " $R$ " " values were found for the whole release process, which indicates a diffusion-controlled release. Diffusion is related to transport of the drug from the dosage matrix into the dissolution fluid, depending on the concentration. As the concentration gradient varies, the drug is released, and the distance for diffusion increases. This could explain why the drug diffuses at a comparatively slower rate as the distance for diffusion increases, which is referred to as the square-root kinetics or Higuchi's equation. In this experiment, the in vitro release profiles of the drug from all the formulations could be best expressed by Higuchi's equation, as the plots showed high linearity $\left(\mathrm{R}^{2}=0.97\right.$ to 0.99$)$. To confirm the diffusion mechanism, the data were fitted into Korsmeyer's equation. Almost all the formulations showed good linearity $\left(\mathrm{R}^{2}=0.98\right.$ to 0.99$)$, with diffusional exponent (n) values ranging from 0.44 to 0.89 , indicating that diffusion was the dominant mechanism of drug release, with these formulations indicative of quasi-Fickian diffusion (case I transport). The diffusional exponent of batches indicates non-Fickian type of release mechanism i.e., 
drug release is by coupling of Fickian diffusion and polymer matrix relaxation-and so called anomalous diffusion-and may indicate that drug release is controlled by more than one process.

Table 2. Physical properties of betahistine dihydrochloride granules (F-1 to F-6)

\begin{tabular}{ccccccc}
\hline $\begin{array}{c}\text { Formulati } \\
\text { on }\end{array}$ & $\begin{array}{c}\text { Loose bulk density } \\
(\mathrm{LBD})(\mathrm{gm} / \mathrm{ml})\end{array}$ & $\begin{array}{c}\text { Tapped bulk density } \\
(\mathrm{TBD})(\mathrm{gm} / \mathrm{ml})\end{array}$ & $\begin{array}{c}\text { Carr's Index } \\
(\%)\end{array}$ & $\begin{array}{c}\text { Hausner } \\
\text { ratio }\end{array}$ & $\begin{array}{c}\text { Angle of repose }\left({ }^{0}\right) \\
\text { Moisture content } \\
(\%)\end{array}$ & $\begin{array}{c}\text { (\%) } \\
\text { F-1 }\end{array}$ \\
\hline F-2 & 0.329 & 0.394 & 16.50 & 1.20 & 27.92 & 1.0139 \\
F-3 & 0.312 & 0.37 & 15.68 & 1.19 & 25.63 & 0.9632 \\
F-4 & 0.329 & 0.407 & 15.48 & 1.18 & 29.07 & 1.0033 \\
F-5 & 0.339 & 0.392 & 16.07 & 1.19 & 25.94 & 0.9304 \\
F-6 & 0.351 & 0.412 & 17.72 & 1.22 & 27.62 & 0.9262 \\
\hline
\end{tabular}

Table 3. Physical properties of betahistine dihydrochloride matrix tablet (F-1 to F-6)

\begin{tabular}{cccccc}
\hline Formulation & Average weight $(\mathrm{mg})$ & Diameter $(\mathrm{mm})$ & Thickness $(\mathrm{mm})$ & Hardness $(\mathrm{N})$ & Friability $(\%)$ \\
\hline F-1 & $99.4 \pm 0.17$ & 6.5 & $3.97 \pm 0.01$ & $36.7 \pm 0.2$ & 0.24 \\
F-2 & $100.3 \pm 0.11$ & 6.5 & $3.93 \pm 0.02$ & $39.1 \pm 0.4$ & 0.31 \\
F-3 & $99.7 \pm 0.15$ & 6.5 & $3.90 \pm 0.02$ & $38.9 \pm 0.6$ & 0.25 \\
F-4 & $99.1 \pm 0.08$ & 6.5 & $3.92 \pm 0.01$ & $38.6 \pm 0.2$ & 0.21 \\
F-5 & $101.5 \pm 0.09$ & 6.5 & $3.89 \pm 0.01$ & $39.5 \pm 0.4$ & 0.22 \\
F-6 & $98.9 \pm 0.12$ & 6.5 & $3.86 \pm 0.01$ & $39.3 \pm 0.3$ & 0.30 \\
\hline
\end{tabular}

Table 4. Percentage (\%) of release of nine formulations (F-1 to F-6) of Betahistine Dihydrochloride tablets

\begin{tabular}{ccccccc}
\hline Time (hrs) & \multicolumn{7}{c}{ Cumulative amount of drug released (\%) } \\
\cline { 2 - 7 } & F1 & F2 & F3 & F4 & F5 & F6 \\
\hline 0 & 0.00 & 0.00 & 0.00 & 0.00 & 0.00 & 0.00 \\
1 & 10.11 & 13.56 & 8.68 & 16.21 & 12.29 & 8.32 \\
2 & 16.96 & 22.94 & 17.67 & 39.24 & 33.24 & 24.59 \\
4 & 39.98 & 42.72 & 31.19 & 70.12 & 58.97 & 49.73 \\
6 & 52.03 & 57.54 & 45.59 & 83.49 & 70.19 & 63.22 \\
8 & 65.37 & 72.16 & 59.14 & 93.24 & 82.13 & 71.98 \\
12 & 82.13 & 97.03 & 73.51 & 99.89 & 93.28 & 84.61 \\
\hline
\end{tabular}

Table 5. Interpretation of R-square values and rate constants for different release kinetics of six formulations (F-1 to F-6)

\begin{tabular}{|c|c|c|c|c|c|c|c|c|c|c|}
\hline \multirow[t]{2}{*}{ Formulation } & \multicolumn{2}{|c|}{ Zero order } & \multicolumn{2}{|c|}{ First Order } & \multicolumn{2}{|c|}{ Higuchi } & \multicolumn{2}{|c|}{ Korsmeyer-Peppas } & \multicolumn{2}{|c|}{ Hixson-crowell } \\
\hline & $\mathrm{R}^{2}$ & $\mathrm{~K}_{0}$ & $\mathrm{R}^{2}$ & $\mathrm{~K}_{1}$ & $\mathrm{R}^{2}$ & $\mathrm{~K}_{\mathrm{h}}$ & $\mathrm{R}^{2}$ & $\mathrm{n}$ & $\mathrm{R}^{2}$ & $\mathrm{~K}_{\mathrm{HC}}$ \\
\hline F 1 & 0.968 & 7.017 & 0.993 & -0.062 & 0.957 & 25.37 & 0.984 & 0.887 & 0.998 & -0.171 \\
\hline $\mathrm{F} 2$ & 0.985 & 7.994 & 0.954 & -0.095 & 0.961 & 28.72 & 0.998 & 0.807 & 0.964 & -0.252 \\
\hline F 3 & 0.977 & 6.269 & 0.995 & -0.049 & 0.956 & 22.55 & 0.994 & 0.872 & 0.997 & -0.141 \\
\hline F-4 & 0.844 & 8.45 & 0.906 & -0.229 & 0.954 & 32.69 & 0.922 & 0.734 & 0.997 & -0.347 \\
\hline F 5 & 0.893 & 7.874 & 0.997 & -0.097 & 0.967 & 29.82 & 0.933 & 0.799 & 0.986 & -0.233 \\
\hline F 6 & 0.914 & 7.32 & 0.995 & -0.069 & 0.959 & 27.28 & 0.938 & 0.928 & 0.980 & -0.186 \\
\hline
\end{tabular}

$\mathrm{k}_{0}=$ zero-order rate constant; $\mathrm{k}_{\mathrm{H}}=$ Higuchi rate constant; $\mathrm{R}^{2}=$ Correlation coefficient 


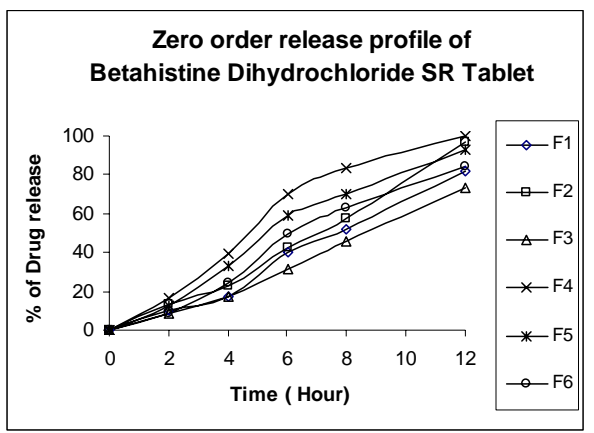

Figure 1. Zero order release kinetics of Betahistine SR tablets

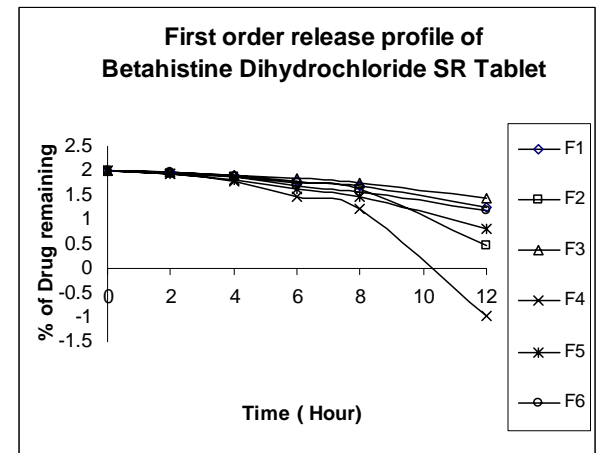

Figure 2. First order release kinetics of Betahistine SR tablets

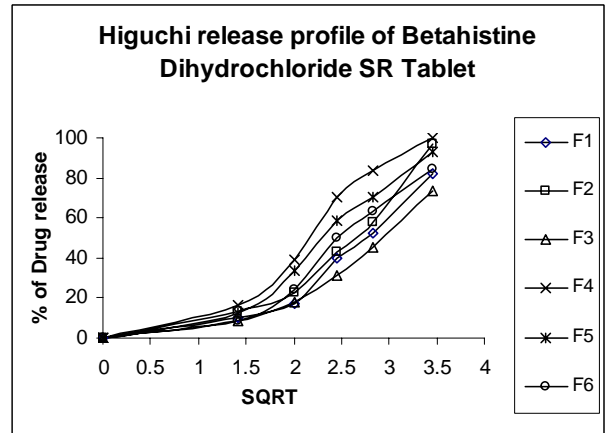

Figure 3. Higuchi release kinetics of Betahistine SR tablets

\section{CONCLUSION}

Betahistine dihydrochloride relieves vertigo symptoms by improving circulation in the microvasculature of the inner ear which leads to a pressure reduction on the membranous labyrinth and relieves the symptoms of Meniere's disease. The experiment revealed that Methocel K4M CR, Methocel K15M CR and Methocel K100 LVCR in varying proportions control the Betahistine Dihydrochloride release effectively for 12 hours; hence the formulations can be considered as a twice daily sustained release tablet of Betahistine
Dihydrochloride which was comparable to theoretical release profile. In most cases the release kinetics of Betahistine Dihydrochloride from the matrix tablets appeared to follow Higuchi and Korsmeyer Pappas equation which indicated that the drug was released from the matrix tablets predominantly by diffusion. From the study it was concluded that the combination of $11.62 \%$ Methocel K4M CR with $34.87 \%$ Methocel K100 LVCR and 11.62\% Methocel K15M CR with $34.87 \%$ Methocel K100 LVCR met the desired sustained release action. Further study on the Betahistine Dihydrochloride sustained release is required to obtain in vivo data of these formulations. The optimized formulations may be used for the development of Betahistine Dihydrochloride sustained release tablet for commercial production.

\section{ACKNOWLEDGEMENT}

The authors wish to thank Incepta Pharmaceuticals Limited, Dhaka, Bangladesh for providing raw materials and also for technical support.

\section{REFERENCES}

1. Beduschi, M.C., Beduschi, R., Oesterling, J.E., 1998. Alphablockade therapy for benign prostatic hyperplasia: from a nonselective to a more selective $\alpha 1$ Aadrenergic antagonist. Urology 51, 861-72.

2. Dutia, M.B., 2000. Betahistine, vestibular function and compensation: in vitro studies of vestibular function and plasticity, Acta otolaryngologica, 544,11-14

3. Dabbagha, M.A., Forda,J.L. , Rubinsteina, M.H., Hogan, J.E., 1996. Effects of polymer particle size, compaction pressure and hydrophilic polymers on drug release from matrices containing ethylcellulose. Inter. J. Pharm. 140, 8595.

4. Cameron, C.G., McGinity, J.W., 1987. Controlled release theophylline tablet formulations containing acrylic resins, II. Combination resin formulations. Drug Dev. Ind. Pharm. 13, 1409-27.

5. Colombo, P., 1993. Swelling-controlled release in hydrogel matrices for oral route. Adv. Drug Delivery Rev. 11, 37-57.

6. Guay, D.R., 2004. Extended-release alfuzosin hydrochloride: A new alpha-adrenergic receptor antagonist for symptomatic benign prostatic hyperplasia. Am. J. Geriatr. Pharmacother. 2, 14-23.

7. British Pharmacopoeia. 2005 London: HSMO 